\title{
Was die geplante Gesundheitsreform für Ihre Praxis bedeutet
}

— Die Bundesregierung will mit dem für 2012 geplanten Versorgungsgesetz die ambulante, wohnortnahe ärztliche Versorgung, vor allem im hausärztlichen Bereich, sichern. Dem Gemeinsamen Bundesausschuss (GBA) soll deshalb vorgegeben werden, die Planungsbereiche so zu gestalten, dass sie einer flächendeckenden Versorgung dienen. Dabei soll bei der Struktur und Größe der Planungsbereiche zwischen hausärztlicher, fachärztlicher und spezialisierter fachärztlicher Versorgung differenziert werden können. Ferner muss bei der Anpassung der Verhältniszahlen die demografische Entwicklung berücksichtigt werden. Der Auftrag an den Gemeinsamen Bundesausschuss, in der Bedarfsplanungsrichtlinie eine Bestimmung über Vorgaben für die ausnahmsweise Besetzung zusätzlicher Vertragsarztsitze zu treffen (Sonderbedarfszulassungen), wird erweitert und sprachlich präziser gefasst.

Alle Leistungen von Ärztinnen und Ärzten, die in strukturschwachen Gebieten tätig sind, sollen grundsätzlich von Leistungsabstaffelungen ausgenommen werden. Die regionalen Vertragspartner erhalten die Option, Preiszuschläge für Leistungen von besonders förderungswürdigen Leistungserbringern, die in strukturschwachen Gebieten tätig sind (z.B. mit höherer Versorgungsqualität) zu vereinbaren.

Um flexibel auch finanzielle Anreize für die Niederlassung in unterversorgten oder drohend unterversorgten Gebieten und bei besonderem Versorgungsbedarf setzen zu können, erhält die Kassenärztliche Vereinigung (KV) die Möglichkeit, über einen neu einzurichtenden Strukturfonds, in den $0,1 \%$ der jeweiligen Gesamtvergütung seitens der KV und ergänzend eine entsprechend gleich große Summe seitens der Krankenkassen einfließen, flexibel und ungebunden gezielte Maßnahmen zu ergreifen. Gedacht ist an Investitionskostenzuschüsse, Vergütungs- und Ausbildungszuschläge oder auch die Förderung von Studenten (Stipendien).

\section{MMW Kommentar}

Interessant sind die Vorstellungen der Politiker im Hinblick auf die Beeinflussung der Ärzteverteilung. Neben der Neuordnung der Bedarfsplanung soll die bestehende Möglichkeit der Kassenärztlichen Vereinigungen, in überversorgten Gebieten den freiwilligen Verzicht auf die vertragsärztliche Zulassung finanziell zu fördern, erweitert werden. Hierzu wird die bisherige Beschränkung der Förderung auf Ärztinnen und Ärzte, die mindestens 62 Jahre alt sind, aufgehoben. Es wird klargestellt, dass auch der Aufkauf einer Arztpraxis durch die KV bei Verzicht auf eine Ausschreibung zur Nachbesetzung eine Möglichkeit der finanziellen Förderung darstellt. Darüber hinaus wird den Kassenärztlichen Vereinigungen ermöglicht, bei der Ausschreibung von Vertragsarztsitzen zur Nachbeset- zung in überversorgten Planungsbereichen ein Vorkaufsrecht auszuüben.

Wie nach geltendem Recht ist das wirtschaftliche Interesse an der Verwertung der Arztpraxis des ausscheidenden Vertragsarztes geschützt. Ein Vorkaufsrecht der Kassenärztlichen Vereinigung besteht jedoch nicht, wenn sich ein Kind, Ehegatte oder Lebenspartner des ausscheidenden Vertragsarztes oder ein Vertragsarzt, mit dem die Praxis bisher gemeinschaftlich ausgeübt wurde, um die Nachbesetzung bewerben. Bewerberinnen oder Bewerber für einen Arztsitz sollen künftig besonders berücksichtigt werden, wenn sie bereit sind, besondere Versorgungsbedürfnisse zu erfüllen wie z.B. die zusätzliche Versorgung in nahegelegenen schlecht versorgten Gebieten.

\section{MVZ-Anzahl steigt weiter}

_ Mit Stichtag zum 30. Juni 2010 gibt es in Deutschland insgesamt 1567 Medizinische Versorgungszentren (MVZ). In Bayern, Niedersachsen und Berlin wurden bislang die meisten MVZ zugelassen. Der überwiegende Teil wurde von Vertragsärzten (47,3\%) oder Krankenhäusern (38,5\%) gegründet. Die bevorzugten Rechtsformen sind die GmbH bzw. die Gesellschaft bürgerlichen Rechts (GbR). In den MVZ arbeiten 7889 Ärzte. 83\% sind als angestellte Ärzte, $17 \%$ als Vertragsärztinnen/ärzte tätig. In einem MVZ arbeiten im Durchschnitt fünf Ärzte. Die Tendenz ist weiterhin steigend. Hausärzte und Internisten sind die Fachgruppen, die in den MVZ am häufigsten vertreten sind.

\section{MMW Kommentar}

Berlin weist mit durchschnittlich 46 Ärzten je zugelassenem MVZ die höchste Dichte je Arzt und mit durchschnittlich 24000 Einwohnern je zugelassenem MVZ die höchste Dichte je Einwohner an MVZ auf. MVZ gründen sich sowohl in städtischen als auch ländlichen Gebieten. Allerdings lässt sich die Mehrzahl der MVZ in Gebieten mit einer hohen Einwohnerzahl und-dichte nieder. Die Anzahl der MVZ insgesamt und auch die Anzahl der MVZ, bei denen ein Krankenhaus als Träger beteiligt ist, steigt stetig an. Auch die Anzahl der angestellten Ärzte in den MVZ steigt seit dem 4. Quartal 2005 stärker an als die Zahl der in den MVZ tätigen Vertragsärzte. Dieser Trend setzt sich auch in den Folgequartalen fort. Dies erklärt sich daraus, dass in den MVZ, die sich in Trägerschaft eines Krankenhauses befinden, Ärzte überwiegend im Anstellungsverhältnis arbeiten. MVZ in Trägerschaft von Krankenhäusern konzentrieren sich dabei stärker in Mecklenburg-Vorpommern, Thüringen, Brandenburg und Schleswig-Holstein. 